\title{
The Knowledge that Matters in Professional Practices
}

Professor Anne Edwards (OSAT, Oxford University Department of Education)

Professor Harry Daniels (CSAT, University of Bath)

Addresses:

Professor Anne Edwards

OSAT

OUDE

15, Norham Gardens

Oxford OX2 6PY

anne.edwards@education.ox.ac.uk

Professor Harry Daniels

CSAT

Department of Education

University of Bath

Claverton Down

Bath BA2 7AY

h.r.j.daniels@bath.ac.uk

Anne Edwards is Director of the Department of Education at the University of Oxford, where she co-convenes the Oxford Centre for Sociocultural and Activity Theory Research. She writes extensively on professional learning and relational forms of expertise. She is a visiting Professor at the University of Oslo and Kansai University.

Harry Daniels is Director of the Centre for Sociocultural and Activity Theory research at the University of Bath. He draws on Vygotskian and Bernsteinian theory to study processes of marginalisation, exclusion and collaboration and has written extensively on Vygotsky's contribution to education. He holds visiting professorships at Griffith University, Kansai University and Moscow State University of Psychology and Education. 


\title{
The Knowledge that Matters in Professional Practices
}

\author{
Anne Edwards (University of Oxford) and Harry Daniels (University of Bath)
}

\begin{abstract}
We draw on the analytic resources of cultural historical activity theory (CHAT) and the work of (Basil) Bernstein and Knorr Cetina to examine evidence from a study of inter-professional practices in children's services in three English local authorities (local government systems). The study traced the horizontal (e.g. cross service) and vertical (e.g. between strategy and operation) integration of services for children including social work and educational psychology at time of reductions in local authority funding. Interviews and meetings, structured by the principles of Developmental Work Research (DWR) (Engeström 2007), required senior local authority staff to identify the objects of activity that shaped the work of the services for which they were responsible, to reveal how those objects were threatened by changing configurations of services and to reflect on what features of the services should be taken forward in these reconfigurations. The study therefore made visible the motives that become evident when professional knowledge is brought to bear on problems of professional practices. In examining the interplay between professional knowledge - which includes notions of the service ideal - and objects of activity we identified 'what matters' (Edwards 2010) in practices within services and 'what matters' when practices intersect and objects of activity are negotiated across practice boundaries.
\end{abstract}

\section{The knowledge that matters in professional practice}

What being a professional entails has been changing, not simply because of a broadening of the category, but also because of the impact of the institutional conditions in which professionalism is enacted. Almost 50 years ago Wilensky, criticising the then increasing bureaucratising of professional practice, reminded his readers of the importance of the professional values and motive-led decisionmaking that he believed were consequently being eroded.

...the degree of professionalization is measured not just by the degree of success in the claim to exclusive technical competence, but also by the degree of adherence to the service ideal and its supporting norms of professional conduct.

(Wilensky 1964: 141)

The importance of the service ideal and the influence of supporting norms are also central to the arguments we shall present about professional practice in the public sector. Like Wilensky we attempt to call attention to what makes professionals distinctive by pointing to how professionalism is constructed in practices. Reflecting current concerns with knowledge in practices, we shall argue for attention to the intertwining of motives in professional practices with the knowledge that matters in professional work and the structuring norms to be found in the organisations inhabited by professionals.

The concerns identified by Wilensky have not been reduced by more recent changes in the organisation of professional work in the public sector. The 1980s' and 90s' development of new public management (NPM) systems in the public sector (Hood 1991; Hood and Peters 2004), with their emphases on accountability, standards, performance management and outputs, not only disrupted the picture painted by Wilensky. But in so emphatically shifting attention from norms and 
procedures to targets and outcomes, NPM was guilty of paying too little attention to professional knowledge and practices and how they are structured and developed.

Elsewhere Edwards has argued (Edwards 2010) that we need a refreshed version of being a professional in the public sector, which sustains the service ideal and emphasises knowledge in practices as a resource to be recognised and deployed in work on complex problems. We now attempt to contribute to that refreshed version by working with the intellectual legacies of Lev Vygotsky, Karen Knorr Cetina and Basil Bernstein to examine how the knowledge that matters in professional practice arises in a dialectical relationship between practitioners and work practices within institutional settings.

\section{The professional in practices}

Our starting point for identifying the knowledge that is valued in professional work is to see practices as historically formed, imbued with knowledge, freighted with emotion and shaped by the values and purposes of the institutions in which they are located. It is in these emotionally freighted practices that the identities that mediate professionals' engagement with both professional knowledge and with clients are formed. How practitioners work with knowledge and with clients is therefore filtered by what is seen as of professional value and as possible to accomplish.

From this perspective, which owes a great deal to Vygotsky's legacy in the form of cultural historical activity theory (CHAT) (Cole 1996), the knowledge in use in professional practice is selected and organised according to the motives that shape practice. Professional work therefore involves an aligning of personal motives, such as keeping children safe, with the motives to be valued within the practices inhabited by the professionals such as the service ideal. This alignment is never straightforward for the individuals or for the practices, which are themselves shaped and reshaped in response to changing social conditions.

A CHAT perspective on these fluid situations would describe them as negotiations of activities and outcomes with other practitioners, and with clients, in value-laden practices. A CHAT analysis is therefore an attempt to get beyond descriptions of role and expert knowledge in shifting work systems, to focus instead on the decision-making, acting and orchestration of work on complex problems which change as they are worked on. Barley and Kunda (2001), from an organisational science perspective, have pointed to the need for this kind of approach to understanding work and its conditions:

In everyday life roles are dynamic and behavioural; not only are their components negotiated and renegotiated in the flow of activity, but over time old roles disappear and new ones emerge.... What is required is a conceptualisation of role that emphasises action and interaction and that also articulates with an image of organisational structure in an empirically specifiable manner.

(Barley and Kunda 2001: 89)

The task is not an easy one: it calls for linking the agency of the practitioner with the motives of the practice while considering the implications for the institutions in which these practices are located. We are proposing that CHAT alone cannot meet this challenge. Rather the analytic tools offered by 
by Knorr Cetina and Bernstein assist, in turn, in revealing how these emotions are played out in the ways in which professionals engage in and with knowledge in their work; and connecting analyses of action in activity in practices with analyses of the structuring and emotional power of discourses in organisations.

The power of socially created motives in cultural historical analyses is seen clearly in the groundbreaking work of Hedegaard on children's development. There she argues that to understand people's development into functioning members of their communities we should begin by examining what is expected of them. As people learn they engage in a world where artefacts and ideas are already invested with meanings that are culturally specific, therefore development should be seen 'as demands from the world onto the person.' (Hedegaard in press). Here we see that what matters is mediated through engagement in practices with others where culturally-specific motives shape what is possible to accomplish.

The argument is that the motives which guide social action in situations are formative in the generation of meaning for the actor and the observer. Consequently, changing the social situation of action can bring a change in motive which in turn transforms the meaning of actions that may, on first observation, appear identical. For example, the meanings ascribed by a teacher to an assessment of a child's work may differ across a conversation with teacher colleagues and a discussion with the child's parent.

Alongside Vygotsky's desire to offer a non-dualist account of the relationship between affect and cognition, he has encouraged us to try to understand the intertwining of action, emotions and motive in human activity. Motives in his legacy therefore always retain a notion of social formation. Motivation is, in contrast, a more profoundly individualist conception. In the argument that follows we distinguish between personal motivations and the motives that orient and drive activities or practices and people's engagement in them and focus on the latter.

Hedegaard's analysis of the dialectic between person and motive-laden practices owes a great deal to that of A.N. Leont'ev who was a close colleague of Vygotsky's in Moscow in the late 1920s. We too will draw on Leont'ev's notion of motive in our discussion of what knowledge matters in professional work. The central concept here is the object of activity.

Leont'ev tried to overcome the dualism of contemporary mainstream psychology by weaving together motives, goals and social conditions. His argument was that 'society produces the activity of the individuals forming it' (1978a: 7). A key to this dialectic between people and activity in society was the object of activity, which gives rise to the object motive, which directs the participation of the actors in activities. He explained:

The main thing that distinguished one activity from another, however, is the difference in their objects. It is exactly the object of activity, that gives it a determined direction. According to the terminology I have proposed, the object of activity is its true motive.

(Leont'ev 1978a: 17)

For Leont'ev the object motive, i.e. what is recognised by actors as what matters in an activity, arises in a dialectic between person and the potential object of activity. In the study of inter-professional 
working that we shall discuss later we found that an object of activity for several professionals might be a particular child's trajectory; but how different practitioners worked on that trajectory would be mediated by what their professional backgrounds allow them to recognise and respond to it. For example, the 'determined direction' of a social worker would be different from that of a teacher and each would act accordingly. The agency of the professional is therefore an important element; we are describing a dialectic and are not working with a view of practitioners being swept mindlessly along through their engagement in practices which shape their actions.

The agency of practitioners can therefore give rise to new knowledge. Like Orlikowski $(2002 ; 2006)$, we take a practice view of knowledge when examining professional work, we see professional knowledge as embedded in these practices and as 'not static or given, but as a capability produced and reproduced in recurrent social practices' and potentially at least 'always in the making' (Orlikowski 2006: 460). It is a view which chimes with Leont'ev's admiration for the connection that Marx made between cognition and practice.

A profound revolution brought about by Marx in the theory of cognition is the idea that human practice is the basis for human cognition; practice is that process in the course of whose development cognitive problems arise, human perceptions and thought originate and develop, and which at the same time contains in itself criteria of the adequacy and truth of knowledge...

(Leont'ev 1978b: 2)

However, Leont'ev's own development of the idea of the object of activity takes Marx's focus on practices much further. It recognises that objects of activity exhibit a motivating force which actors might recognise in different ways; and reminds us that the relationship between acting subject and object of their activity is never direct, but is always mediated by the motives in a practice.

So far our discussion appears to assume that professionals are able easily to exercise professional agency in practices that recognise and value the service ideal. However, we know it is not always the case. The distinction made by Evetts between 'organizational professionalism' and 'occupational professionalism' (2009: 248) in her 2009 discussion of how the professions are changing in relation to the constraints of NPM, is therefore useful. The former is characterised by a discourse of professionalism as a form of occupational control within hierarchical structures, and is a feature of NPM in public service sector work. The latter emphasises relationships over structures, and demonstrates an orientation to work where 'the needs and demands of audiences, patients, clients, students and children are paramount' (2009: 252).

This distinction is central to our concern that in CHAT too little attention is paid to what knowledge is recognised, valued and brought into play to work on objects of activity. We suggest therefore that CHAT analyses of motives in practices may be usefully augmented by two strands of work: (i) following Knorr Cetina, that we need to be alert to the emotional aspects of engagement with knowledge in practices that are not routine; and (ii) drawing on Bernstein, that the emotional features of different institutional forms give rise to different forms of professional practices, values and orientation.

Engaging with the knowledge that matters 
Knorr Cetina's work on epistemic cultures (1999) brings the knowledge that matters in practices to the fore. Her notion of 'engrossment' with knowledge can illuminate those professional practices which are not merely a matter of rule-following but involve engaging with what is valued professionally. The idea of engrossment, elaborated in her study of how traders in investment banks work with the market as an object of 'attachment' (Knorr Cetina and Brueggar 2002) is therefore helpful. There the market is seen as an object of knowledge which is unfolding and engaging those who work on it, not just to understand it, but also to test, move and manipulate it and be stimulated by its capacity to generate questions. Engagement arises through practitioners' need to continually work on the object of knowledge and define it. Knorr Cetina and Brueggar also describe this engagement in terms of 'lack' or incompleteness in the traders' understanding of it and their desire to pursue a better grasp of what is there.

She suggests that the epistemic knowledge arising through engrossment offers potentially stabilising effects for the experts who seek a relationship with the knowledge that is embedded in what they do. In an earlier paper, discussing what she termed 'epistemic' or knowledge objects she explained:

...objects serve as centering and integrating devices for regimes of expertise that transcend an expert's lifetime and create the collective conventions and the moral order commutarians are concerned about. Object worlds also make up the embedding environments in which expert work is carried out, thus constituting something like and emotional home for expert selves.

(Knorr Cetina 1997: 9)

Here she is not describing knowledge objects as instruments which are 'ready to hand' (p. 10): these should be seen as tools. Knowledge objects, in contrast, are problematic and need to be worked on to refine them, rather than worked with on routine tasks. Objects of knowledge, she explains are 'the goal of expert work' (p. 12), where the expert self becomes bound to the knowledge object through a sense of 'lack' and the creation of a 'structure of wanting' ( $p$ 16) which sustains the connection. The outcome is a 'state of subjective fusion with the object of knowledge' (p. 18). In CHAT terms, the self and the knowledge that matters become intertwined, informing identities which mediate professional actions. What CHAT adds to Knorr Cetina's analyses is that these actions are in response to the object motive i.e. what is recognised and worked on in practices. The interplay between the knowledge that matters in professional practice and the interpretations of the problem to be worked on is therefore surfaced.

Knorr Cetina's work also offers a way of understanding how knowledge as a tool in practices is made and remade that is not easily apparent in CHAT analyses. Knowledge does not get forgotten in CHAT: the importance of mediation of what matters in a culture, through the use of cultural tools such as language intertwined with material artefacts such as assessment forms, means that knowledge is a central element in the theory. But the ideas about epistemic objects as unfolding entities that engage the self and which are open to scrutiny as they are worked with in knowledge-rich practices, which come from Knorr Cetina's work, are useful when examining how professions take up tools and engage with them as 'ready to hand' or as potentially unfolding entities that offer increasing scope for professional decision-making . 
CHAT analyses do recognise that interpreting a professional task, and manipulating resources to work on it, are actions which are imbued with professional knowledge, which includes values. In responsive professional work CHAT 'objects of activity' such as a child's trajectory are also marked by their unfolding nature as they are worked on by practitioners who use tools in open -ended ways so that the object of activity's complexity is increasingly revealed (Edwards et al. 2009). As this happens, professionals are pulled forward in their work by' what matters such as commitment to the ethics of care. However, the focus in CHAT is not the knowledge in use; rather it is problem being worked on with the knowledge in use. We suggest that CHAT analyses that attempt to unravel what makes Evetts' occupational professionalism so distinctive are augmented by attention to how professionals engage with knowledge in their work. The particular distinction to draw is between the rule-bound engagement with the 'ready to hand' that Knorr Cetina dismisses as simply 'tools', and the knowledge objects to which professionals are emotionally tied as they work with and on them to take control over the complex problems they encounter in their work.

\section{Institutional discourses and the knowledge that matters}

We now turn to examining interplays between institutional conditions, practices and the knowledge in use. To do so we call on the distinction that Bernstein made between what he termed the instrumental and the expressive or regulative discourses (Bernstein 2000). The instrumental discourse refers to the transmission of skills and their relation to each other, and the expressive or regulative discourse to the principles of social order, relation and identity. Expressive or regulative discourse therefore communicates an institution's public moral practice, values beliefs and attitudes, principles of conduct, character and manner. It also transmits features of the institution's local history, local tradition and community relations.

This model of discourse provides a response to one of the theoretical demands that have remained unfulfilled in CHAT. It provides a way of theorizing discourse as a tool within Activity Theory that incorporates both instrumental and moral / affective dimensions. Crucially it also allows the analysis and description of the discourse to be related to the relations of power and control that obtain in the setting in which the activity is enacted.

We suggest that these discourses, which are embedded in institutional practices, both structure and are structured by the institution and give rise to the knowledge that can be used to mediate and take forward professional work. Importantly, organisations differ in the relative salience of each discourse with the result that institutional purposes are revealed in professional talk in and about practices. A particular contribution of Bernstein's analyses is therefore his attention to the affective aspects of cognition and action. Following Bernstein, our premise is that differences in discursive practices in organisations relay differences in the relationship between the instrumental and the expressive elements in an organisation, and that these in turn lead to differences in what knowledge is valued in professional practices.

Bernstein's (2000) model was designed to relate macro-institutional forms to micro-interactional levels and the underlying rules of communicative competence. He focused on two levels; a structural level and an interactional level. The structural level is analyzed in terms of the social division of labour it creates (e.g. the degree of specialisation, and thus strength of boundary between professional groupings) and the interactional with the form of social relation it creates (e.g. the degree of control that a manager may exert over a team member's work plan). The social 
division is analysed in terms of strength of the boundary of its divisions, that is, with respect to the degree of specialisation (e.g. how strong is the boundary between professions such as teaching and social work). Thus the key concept at the structural level is the concept of boundary, and structures are distinguished in terms of their relations between categories. The interactional level emerges as the regulation of the transmission/acquisition relation between teacher and taught (or the manager and the managed), that is, the interactional level comes to refer to the pedagogic context and the social relations of the workplace or its equivalent.

The move from the socio- material structural arrangements to the structure of the pedagogic discourse provides a way of attending to the critique of the notion of tool in Vygotskian theory that Bernstein outlined.

The metaphor of 'tool' draws attention to a device, an empowering device, but there are some reasons to consider that the tool, its internal specialised structure is abstracted from its social construction. Symbolic 'tools' are never neutral; intrinsic to their construction are social classifications, stratifications, distributions and modes of recontextualizing.

(Bernstein 1993, p. xvii)

In Bernstein's work power is spoken of in terms of classification which is manifested in category relations which themselves generate recognition rules. Possession of which allows the acquirer to recognise a difference that is marked by a category such as the rules which allow a professional to be recognised as belonging to particular professional group. This is not simply a matter of finding out which service someone belongs to, it also refers to the ways in forms of talk and other actions may be seen to be belonging to a particular professional category or grouping. When there is strong insulation between categories (i.e. social worker, family worker), each category is sharply distinguished, explicitly bounded and has its own distinctive specialisation, then classification is said to be strong. When there is weak insulation then the categories are less specialised and there distinctiveness is reduced; then classification is said to be weak.

Different institutional modalities may be described in terms of the relationship between the relations of power and control which gives rise to distinctive discourses which mediate engagement with the knowledge in use in professional work. For example in children's services, where the beliefs about the knowledge that matters gives rise to a strong classification and strong framing of specialist practices it is expected that there will be a separation of professional discourses and an emphasis upon acquisition of specialised skills which may downplay both inter-professional collaboration and engaging children and families in solving problems. Relations are therefore likely to be clearly hierarchical with the instrumental and the regulative discourses as relatively distinct. However, if beliefs about the knowledge that matters gives rise to a weaker classification and weaker framing of the practice then there is likely to be easier cross-service collaboration and greater engagement of children and families. In this case the regulative discourse is embedded in the instrumental

The language that Bernstein has developed, uniquely, allows researchers to take measures of institutional modality. That is to describe and position the discursive, organisational and interactional practice of the institution based on analyses of differences in discursive practices in organisations which reveal differences in the relationship between the instrumental and the expressive elements in an organisation.

However, Vygotsky was not impervious to the importance of the emotional in how people are able to take action; though his approach was rooted in the Russian notion of perezhivanie which is often 
loosely translated as experiencing. For Vygotsky perezhivanie was the integration of cognitive and affective elements, which always presupposes the presence of emotions. He used this concept in order to emphasize the wholeness of the psychological development of children, integrating external and internal elements at each stage of development providing a "unity" of psychological development in the study of the social situation of development.' (Gonzalez-Rey 2002, p. 136). The resonance of his analyses with those of Bernstein is clear in the following extract from his writings.

"The emotional experience [perezhivanie] arising from any situation or from any aspect of his environment, determines what kind of influence this situation or this environment will have on the child. Therefore, it is not any of the factors themselves (if taken without the reference of the child) which determines how they will influence the future course of his development, but the same factors refracted through the prism of the child's emotional experience" [Vygotsky 1994, pp. 339].

This idea of refraction 'through the prism of the child's emotional experience' has been largely ignored in the development of post Vygotskian theory. The rejection of the cognitive/affective dualism which Vygotsky $(1994,1987)$ announced was not followed by a model within which a unitary conception of thinking and feeling could be discussed and implemented within empirical research. It is with these theoretical perspectives in mind that we approached our study.

\section{The study}

The one year study examined the horizontal and vertical integration of services for children such as social work and educational psychology in three local authorities. Integration of this kind was occurring across children's services in England as an obligatory response to the 2004 Children Act (DfES 2004).

The study built on an earlier examination of inter-professional working among operational staff in children's services (Daniels et al. 2007; Edwards et al. 2009). The earlier study had revealed that practitioners who were working directly with children and families were operating in practices shaped by strong motives; that these motives often became explicit when discussing what mattered for them about a particular case to other professionals; and that the organisational structures in which these practitioners operated were not keeping pace with the changes in the practices they should be supporting. For example, we found examples of 'rule-bending', where practitioners ignored institutional requirements such as referring a child to a psychologist via a school's referral system and instead contacted the psychologist directly. These examples substantiated a claim in the 2008 English Audit Commission report on children's services, that inter-professional practices were developing despite the organisational arrangements in which they were operating. They also revealed quite starkly that these professionals had a strong sense of what mattered in work with children which was at times in tension with institutional expectations.

The study at the centre of the present article was set up, in response to the concerns articulated by the Audit Commission report, to help local authorities recognise and mobilise the knowledge that was in play at the front-line in direct work with families. It recognised that practitioners carved out new ways of working and developed their conceptualisations of practice as they worked alongside professionals from other practices in what we have described as systems of distributed expertise (Edwards 2010).

The professionals we interviewed were all senior staff working in children's services with responsibility for enabling integrated cross-service working and had backgrounds in either education 
or social care. Data collection involved CHAT-based interviews ( $n=15$ in each local authority) and two meetings each of two hours in length in each site, which were structured by the principles of Developmental Work Research (DWR) (Engeström 2007). The interviews and meetings, which were all recorded and transcribed, required senior local authority staff to identify the objects of activity that shaped the work of the services for which they were responsible, to reveal how those objects were threatened by changing configurations of services and to reflect on which specific contributions to be made by each service should be taken forward in these reconfigurations.

The DWR-structured meetings can therefore be construed as sites of intersecting practices aiming at taking forward service integration where 'what matters' for each service was publicly negotiated. Talk in the meetings made visible (i) the motives that became evident when professional knowledge was brought to bear on problems of professional practices and (ii) the professional knowledge in which these motives were embedded. In examining the interplay between professional knowledge and object motives we have identified the knowledge that matters in practices both within individual services and when the practices of services intersect and objects of activity are negotiated across practice boundaries.

Interviews and talk data from the DWR sessions in each local authority were analysed to reveal (i) following Knorr Cetina, how engagement with professional knowledge differs in line with the relative salience of the instrumental and the expressive discourses; and (ii) how Bernstein's embedded discourses permit specific ways of accomplishing what matters in practices. The analysis presented here does not focus on the processes of argumentation i.e. how these discourses were deployed, but on the knowledge in play as revealed in how the objects of professional work are categorised and how these categories are employed in negotiations across professional boundaries. Our assumption has been that inter-professional discussions where 'what matters' is both defended and negotiated into reconfigured practices allow analyses of the extent to which the knowledge used is either instrumental or expressive and how each creates different knowledge-informed interpretations of the object of activity for the professionals involved.

\section{Describing the sites and analysing the data}

We shall take just two of the three sites and examine the relationships between discourses; how they permit specific ways of accomplishing what matters in practices; and how engagement with professional knowledge differs according to the relative salience of the instrumental and the expressive discourses.

Smalltown was a new unitary authority which had been carved out from a larger shire county little over a year before we started the study. It was therefore assembling its children's services from scratch. There was a strong demarcation of boundaries between the different professional sectors that comprised children's services. Knowledge travelled efficiently up and down the levels in each professional sector, but not horizontally across sectors. The division of labour was strong with strong classification and framing of practices. There was evidence in the interviews of emotional strains for a number of individuals, but there was little evidence of the expressive being emphasised in institutional discourse.

Largecounty was a long-established shire county in which services functioned in separate geographical areas of the county. Both the previous and the current Director of Children's Services (DCS) recognised that the comfortable laissez-faire operation of services needed revision. 
Nonetheless, the current DCS was at pains to emphasise the expressive while at the same time creating new systems that ultimately called for new rules in an attempt to keep the instrumental and expressive in a productive tension. At the time of the study the DCS was trying to set up a multiprofessional system that called into question both professional boundaries and hierarchies.

\section{The analytic resources from Knorr Cetina}

Knorr Cetina's work alerted us to seek out both what was the knowledge that mattered and how it was engaged with. The expectation was that in some circumstances conversations about work in the DWR sessions and in the interviews would reveal objects of professional knowledge which were seen in Knorr Cetina's terms as unfolding and engaging those who work on them, not just to understand them, but also to test them and pursue the questions they raised. Such engagement takes practitioners beyond compliance with the ready to hand rules of professional behaviour towards a questioning and reshaping of practices and engagement with knowledge-making in professional practice.

\section{The analytic resources from Bernstein}

Bernstein's work provided us with a language of description which allowed a reading of the three local authorities as they did their tacit psychological work through their discursive practices. We needed to be able to describe the most likely forms of institutional practice that would be sustained in those settings i.e. the pedagogic modalities of the settings in which we were intervening.

A crucial element in the description was an attempt to try and identify points at which the communicative action that occurred in the DWR sessions would engage with the transformation of the institution and to recognise how the social structures of the institution impacted on the interactions between the participants and what was the knowledge that appeared to matter for them. From a Bernsteinian standpoint the relations of power and control, which regulate social interchange, give rise to specialised principles of communication. These mediate social relations and shape both thinking and feeling about practices and their purposes.

In previous work we have also recognised the importance of developing an approach to the analysis and description of our research sites that could be used to monitor changes that took place over the course of our interventions (Daniels 2010). These understandings have led to an account of institutional structures as cultural historical products (artefacts) which play a part in implicit (Werstch, 2007) or invisible (Bernstein, 2000) mediation. In the present study, however, we were not attempting to take forward a change programme but to help to reveal the knowledge distributed across children's services in three local authorities.

Within the DWR sessions, and the interviews that provided the data for the sessions, we examined both the horizontal distinctions between professions and the vertical distinctions that marked hierarchies within professions. The former involved assessing the strength of classification (the division of labour between different professions) in the practices of professional agencies and control or framing of the membership of these groups. The latter involved judging the strength of distinctions in the vertical division of labour, the strength of the marking of hierarchy and the associated relations of control within this hierarchy was also seen to be a central facet of the structuring of the DWR sessions. The strength of control over the regulative practice (matters of order, identity and relation) was also noted.

\section{Knowledge in use}




\section{Smalltown}

The extract that follows is from a DWR session in Smalltown which shows that when the expressive is allowed to become salient it can lead to an engagement with what matters. The extract comes after a long discussion of the emphasis on rules and accountability within the local authority which was limiting professionals' freedom of movement. At the point the extract starts a contributor moves from a discussion of the instrumental rule-governed hierarchy of the authority to offer a highly charged expressive account of what they are experiencing which breaks through the account of hierarchical system they were inhabiting. This intervention releases the participants to work towards a recognition of the need to make explicit the motives that were shaping their practices as professionals. The contributors were all senior practitioners within children's services.

A: I think in terms of a family if you looked at this as a family, I think Children's Services is probably the teenager of the council (i.e. the elected body that employs these practitioners).

B: I would say... I was actually thinking along the lines of that analogy and I was going to say the step-child actually, that's how it feels at times.

C: The slightly stroppy and surly step-child who has come to visit.

D: And actually if you want to carry that analogy to its furthest extent, you know, there's certain part of, the centralised part of... the central support section of the council that you could akin to an extremely authoritative paternal figure. I mean...

E: And you could take that even further and suggest that, you know, when there's decisions made on what to finance, do you finance the clothes or do you finance the roof over their head or what do you finance. You're we're severely at risk of just financing the underpants and the socks and not financing the books.

C: If Children's Services is the teenager of the council, what do teenagers do? Take no notice of their parents.

A: Okay, and how do parents respond to that thought, $\mathrm{F}$ (the most senior person there)?

F: Well yeah, yeah, but there's the inherent tension isn't it. Parents will understand that teenagers can make mistakes, they have to learn from their mistakes and there will be that tension. So you don't expect there to be... it's not always going to be rosy is it? And part of learning to live within that family and the family dynamics change, so you would expect that in some senses, that's not a negative... you know, that could be a positive thing if you see it in that way, you know, they don't always know best and I think I know better than you. You probably don't always know best actually, but you have to have the opportunity to test it out. And that's a healthy tension is where I was going with it, it's not necessarily a negative tension.

B: The reason why l'd kind of thought about the concept of a step-child is, you know, when a family is reconstituted and you've got children from two different families joining together in one unit, changes have to be made on both sides. Whereas actually 
at the moment I think there's an expectation that one side will change and the other will not. That's the friction for me, that's the pressure.

Once the expressive was allowed to dominate over the instrumental, and indeed to label in B's last turn, there was a shift to discussing the knowledge that mattered and their need to engage with it.

B. The problem we've got is it requires an altruistic approach to this, which is focused on making the lives of children and families better for the next 20 years. Trying to get a council to have that is quite a different approach isn't it? But it shouldn't stop us as leaders in the organisation for children putting that case forward. At least, you know, at least putting the council in a position where they've got to publicly consider that issue and make some difficult decisions around it. And we might have to live with things we don't like, but at least we've tried to put a case forward.

D. ... we talked earlier about, the importance of prevention, the importance of, you know, providing a range of services at different points in people's lives which are aimed at sustaining them and sustaining families and so on. And yes of course there's always going to be crises, but that's sort of... we've got to make the case out why that's important. We've probably got to think about what value we bring to the council perspective.

\section{Largecounty}

Here participants struggled in order to keep both the instrumental and expressive in play as they identified what matters while planning the implementation of a series of locally based multiprofessional groupings of preventative services across the county. In the first extract, from an interview, there are echoes of Smalltown's interaction in the DWR. Talking of the laissez-faire emphasis on the expressive in the past, one interviewee said.

A.I think we were the naughty child of the corporate centre because I don't think we got corporate at all.

The move from the expressive to a tension between the expressive and the instrumental was a focus of the work underway as another interviewee explained.

B. I think communication on the ground is very often as good as you wish it to be, it's adopting formal protocols that make it happen in a way which is delivering what you want I think is sometimes the challenge.

Evidence of a productive tension between the expressive and the instrumental that was ultimately achieved wove its way through the DWR sessions. Here one participant points to the affective aspects of work on complex problems.

And I think what's happened is, it's about sharing out of responsibilities. Because everybody has seen everybody coming to the table and that gives you confidence, you're not on your own dealing with this, it is part of that Team Around the Child team with that family. Everybody has got responsibilities, everybody will go away and do their jobs and bring it back and you're not on your own. And that confidence is hugely 
important when you are dealing with very complex family situations that you don't feel you're isolated and on your own.

At the same time the instrumental concern with order and process was there in the same DWR session. In this extract we also see the creation of knowledge objects with which people can engage, begin to position themselves in relation to.

We're closing both the service consultation and the individual structure consultation. And I suppose that's the point when we can come back out with how we're going to make this live to people at different parts ... So working on induction and working on the training and development plan for how this is going to operate and how do we speak to different people. And I think the use of practice specific examples can both allay concerns, can give information about actually a way of working, and this is the way we're going to be working ... So I think people will be able to get... you know, the academic people will be able to see that the structure and the research is behind it; people who are practitioners will be able to see, well how will I be working, and then hopefully stakeholders can see that too.

Here the most senior practitioner summarises where they have got to with setting up the new system. The attention to the expressive has led to an overwhelming response to the quite dramatic changes being planned and a willingness to engage with the professional challenges of the new work including working on new practices as knowledge objects in their own right.

All we've seen is it appearing like a magnet and people saying, I want to get involved in that, that's really interesting and I can see how that's going to work, I can see how that works for me and I can see how that will work for other people. Hence the fact that the task and finish groups we're having to ask people not to be on them we've just got so many volunteers there is danger of it being total dysfunctional by the sheer size. And, you know, there's a lot of people feeling quite upset about not being involved in everything that's going on, it's been quite hard to manage. But I think, you know, we have to take heart from that. That's actually genuine interest in being involved in, you know, being part of something that they think has got legs to be really successful.

\section{Discussion}

We have presented two sets of extracts from two local authorities and recognise that we are making large claims from them. However, we have seen throughout our work with children's services (Daniels 2010; Edwards et al. 2009) that a productive tension between instrumental and expressive discourses frees up professionals to engage with the knowledge that matters.

What matters is not necessarily research-based knowledge that was referred to quite frequently in Largecounty; it may be the need to reconfigure a practice to better serve the needs of a client group or a change in roles and responsibilities to be more responsive to client needs and, as we have seen, these too were evidenced in Largecounty. Miettinen and Virkkunen (2005) making links between CHAT and epistemic objects and focusing on their activity theory concerns with organisational change, have argued that practice itself should be treated as an epistemic object and in particular 
practitioners should focus on the institutional contradictions that become evident in it as practices need to change.

However, where the instrumental discourse dominates over the expressive, as was the case in Smalltown, an opportunity to work with the expressive produces a better interplay between the instrumental and expressive permitting a move towards engaging with the knowledge that matters in professional practices.

There has been a recent resurgence of interest in the more affective aspects of development among those who draw on Vygotsky's legacy (Hedegaard, Edwards and Fleer in press). These link motive, emotion and cognition in ways which build on Leont'ev's seminal work. Lompscher, for example, explains the cultural historical conception of motive and its connection to cognition as follows.

Objects which an individual represents cognitively and which satisfy a certain need therefore become emotionally significant for the individual. As such they become the real motive of a concrete goal-oriented, object-determined activity. In this sense, motives represent a unity of cognition and emotion.

(Lompscher 2000: 79)

Vygotsky and his co-workers, notably Leont'ev, saw the societal formation of motives as central to a thesis of the cultural historical formation of mind. A weak point in the work that has taken forward their legacies has been analyses of how institutions mediate societal motives, how they stand between society and the person in institutional practices. Although present in the latter stages of Vygotsky's writing, relatively little attention has been paid to the development of a non-dualist account of cognitive and affective features of human functioning and these relate to the ways in which motives and goals arise in particular situations. We hope that by combining CHAT analyses of work in practices with Bernstein's attention to the structuring power of embedded institutional discourses, and in particular the interplay between cognition and affect which is so central to it, will be an important addition to the CHAT repertoire. In doing so in the context of professional practices oriented towards the wellbeing of vulnerable children we are doing so in ways that points to a refreshed way of considering the service ideal.

By also paying attention, following Knorr Cetina, to the knowledge that arises and matters in professional practices in settings where the old rules which have shaped discrete practices are no longer viable, we hope to make visible the problematic nature of knowledge in practices and how this is intertwined with the motives in practices and practitioners' emotional engagement with knowledge. The intention is that knowledge is not simply regarded as a given embedded in the material artifacts such as assessment forms but is, potentially at least, recognised as always in the making and engrossing. One of Vygotsky's concerns was to better understand the connections between culturally embedded cognition, emotion and intention. By bringing together two analytic resources, not often employed within CHAT research, we hope we have contributed to the task he set up.

We also hope that the implications for organisations which employ professionals are clear. Our conclusion is that there is a need to ensure that the instrumental discourse does not dominate, but 
instead there is a tension between the instrumental and the expressive discourses which permits attention to the knowledge that matters for professionals in their work with clients. Our theory-led qualitative analysis receives support from the findings of Glisson and Hemmelgarm 's (1998) quasiexperimental, three year study of the services provided to 250 children by 32 public children's service offices in 24 counties in Tennessee. The study reported that organisational climate was the primary predictor of children's improved psychosocial functioning and a significant predictor of service quality. In contrast, strong organisational coordination had a negative effect on service quality and no effect on outcomes.

We therefore return, in conclusion, to the distinction made by Evetts between 'organizational professionalism' of NPM and the 'occupational professionalism' represented by what Wilensky termed the service ideal. In England the disruption of rules, roles and systems arising from the policies of the coalition government which set up in May 2010 was exemplified in the November 2010 Schools White Paper. The policy it outlined stripped out the supports of NPM leaving a new freedom for professionals in the service professions. Here the local authorities' role in Education is outlined.

In order to help local authorities deliver this strategic role in the new system we will give them progressively greater freedom, by stripping away targets, rules, regulations and ring-fencing.

(DfE 2010:5.9)

However that freedom brings challenges to professionals and the organisations in which their practices have developed. One DCS summarised the ensuing situation as follows.

The world has changed...(you need to) think about outcomes for families...but sometimes you give people the freedom and they don't know what to do with it.

The opportunity for a refreshed version of professionalism within the English welfare professions has come at a time of diminishing budgets where 'what matters' or the 'why' of practices needs to be to the fore in any decision-making at both institutional and individual levels. Our argument is that this can be achieved by promoting a combining of the affective with the cognitive in professional practice and establishing work systems which make visible the purposes of institutional practices offering opportunities for a dialectic which recognises the engaged expertise of knowledgeable professionals.

\section{References}

Audit Commission (2008). Are we there yet? Improving Governance and Resource Management in Children's Trusts, Local Government National Report, London: Audit Commission.

Bernstein, B. (1977) Class, Codes and Control Vol. 3.: Towards a Theory of Educational Transmissions: 2nd revised edition London: Routledge \& Kegan Paul

Bernstein, B. (2000). Pedagogy symbolic control and identity. Lanham Maryland: Rowman and Littlefield.

Cole, M. (1996). Cultural psychology: a once and future discipline. Cambridge, Mass: Harvard University Press. 
Daniels, H. (2010). The mutual shaping of human action and institutional settings: a study of the transformation of children's services and professional work, The British Journal of Sociology of Education 31(4), 377-393.

Daniels, H., Leadbetter, J. \& Warmington, P. (2007). Learning in and for multi-agency working, Oxford Review of Education, 33 (4), 521-538.

DfES (2004). The Children Act. London: HMSO.

DfE (2010). The Importance of Teaching: the Schools White Paper. London: DfE.

Edwards, A. (2010). Being an expert professional practitioner: the relational turn in expertise. Dordrecht: Springer.

Edwards, A., Daniels, H., Gallagher, T., Leadbetter, J. \& Warmington, P. (2009). Improving interprofessional collaborations: multi-agency working for children's wellbeing. London: Routledge.

Engeström, Y. (2007). Putting Vygotsky to work: the change laboratory as an application of double stimulation. In H. Daniels, M. Cole, \& J.V. Wertsch (Eds.) The Cambridge companion to Vygotsky (pp. 363-382). New York : Cambridge University Press.

Evetts, J. (2009). New professionalism and new public management: changes continuities and consequences. Comparative Sociology, 8, 247-266.

Glisson, C. \& Hemmelgarn, A. (1998). The effects of organizational climate and interorganizational coordination on the quality and outcomes of children's service systems, Child Abuse and Neglect. 22(5), 401-421.

Gonzalez-Rey, F. (2002) L.S. Vygotsky and the Question of Personality in the Cultural-Historical Approach, in D. Robbins and A. Stetsenko (Eds.) Voices Within Vygotsky's Non-Classical Psychology: Past, Present, Future (New York, Nova Science).

Hedegaard, M. (in press). The dynamic aspects in children's learning and development. In Hedegaard, M., Edwards, A. \& Fleer, M. (Eds.) Motives, emotions and values in the development of children and young people. Cambridge: Cambridge University Press.

Hedegaard, M., Edwards, A. \& Fleer, M. (Eds.) (in press). Motives, emotions and values in the development of children and young people., Cambridge: Cambridge University Press.

Hood, C. (1991). A public management for all seasons, Public Administration, 69 (Spring), 3-19.

Hood, C. \& Peters, G. (2004). The middle aging of New Public Management: into the age of paradox? Journal of Public Administration Research and Theory, 4(3), 267-282.

Knorr Cetina, K. (1997). Sociality with objects: social relations in post-social knowledge societies. Theory Culture Society, 14(1), 1-29.

Knorr Cetina, K. (1999). Epistemic cultures: how sciences make knowledge. Cambridge Mass: Harvard University Press. 
Knorr Cetina, K. \& Brueggar, U. (2002). Traders' engagement with markets: a postsocial relationship. Theory Culture and Society, 19 (5-6), 161-185.

Leont'ev, A.N. (1978a). The problem of activity in psychology, in Activity, consciousness and personality. Upper Saddle River, N.J.: Prentice Hall. (downloaded on 29.4.2004 from http:Marxists.anu.edu.au/archive/leontev/works/1978 )

Leont'ev, A.N. (1978b). Marxism and psychological science, in Activity, consciousness and personality. Upper Saddle River, N.J.: Prentice Hall. (downloaded on 29.4.2004 from http:Marxists.anu.edu.au/archive/leontev/works/1978 )

Lompscher, J. (2000). Motivation and Activity

http://www.experiment.lv/rus/biblio/vestnik 7/v7 lomp motivation.htm downloaded 2/12/2009 Miettinen, R., Virkkunen, J. (2005). Epistemic objects, artifacts and organizational change. Organization 12(3), 437-456.

Orlikowski, W. (2002). Knowing in practice: enacting a collective capability in distributed organizing. Organization Science 13(3), 249-273.

Orlikowski, W. (2006). Material knowing: the scaffolding of human knowledgeability. European Journal of Information Systems, 15, 460-466.

Vygotsky, L.S (1987). The collected works of L.S. Vygotsky: Problems of general psychology. (Vol.1). New York: Plenum Press. (Original work 1933-1934).

Vygotsky, L.S (1994). The socialist alteration of man. In The Vygotsky reader (Oxford, Basil Blackwell) pp. 175-184. (Original work 1930).

Wilensky, H. (1964). The professionalization of everyone? The American Journal of Sociology. 70(2), 137-158. 